\title{
Magnetostriction and the Advantages of Using Noncontact Measurements
}

\author{
Setareh Gorji Ghalamestani*, Lieven Vandevelde*, Joris J. J. Dirckx ${ }^{\dagger}$ and Jan A. A. \\ Melkebeek* \\ *Electrical Energy Laboratory (EELAB), Department of Electrical Energy, Systems and Automation (EESA), \\ Ghent University, Ghent 9000, Belgium \\ ${ }^{\dagger}$ Laboratory of Biomedical Physics, University of Antwerp, Antwerp 2020 , Belgium
}

\begin{abstract}
Magnetic noise in electrical machines and transformers are a large portion of the total noise of the device. Part of this magnetic noise is caused by the deformation of the ferromagnetic laminations due to the magnetic field. This effect is called magnetostriction, and it strongly depends on the applied magnetic field, the material properties and external pressure to the material. A strain gauge measurement setup has been applied before to measure the magnetostrictive behaviour of ferromagnetic materials. The results obtained by this setup suffered from some limitations such as the need to filter highfrequency harmonics. Also the measurement results for excitation below $0.8 \mathrm{~T}$ were not easily distinguished from the present noise. Therefore, a new setup using heterodyne laser interferometers has been built. With this new setup, on the contrary to the strain gauge setup, the sample preparation is simple. This new setup and the gradual improvements toward the optimal performance of the setup are presented in this paper.
\end{abstract}

Keywords: Heterodyne laser interferometer,magnetostriction measurements, noncontact measurements PACS: $75.80 .+\mathrm{q}$

\section{INTRODUCTION}

The study of magneto-elastic vibrations of magnetic materials in magnetic cores of electrical machines and transformers has been an interesting issue for researchers in the latest years. Magneto-elastic vibrations, which are also known as magnetic vibrations, cause the major part of the noise in the aforementioned devices. But there are other contributors as well, such as mechanical sources like the bearings, as well as aerodynamical sources, meaning the cooling system. Magnetic vibrations and noise, which are the focus of our research, are produced in two ways which will be explained shortly. Electrical machines and transformers both have a magnetic core which is a stack of laminations of ferromagnetic materials, mostly electrical steels. In electrical machines for instance, the armature current in the stator induces a magnetic induction in the rotor laminations and the interaction of this current and the induced flux density produces force and torque. But at the same time, the alternating nature of this force tries to deform the geometry of the magnetic core, which in turn produces vibrations and leads to the noise. On the other hand, the ferromagnetic material itself deforms when exposed to a magnetic field. This phenomenon is called magnetostriction. So in total, the magnetic forces and magnetostriction together give rise to the magnetic vibrations and noise. Since we can calculate the magnetic forces, the part of the magnetic noise originating from this force can be predicted as well. However the behaviour of magnetostriction is very complicated. It varies for every material and it depends on the magnetization. Therefore, it is impossible to calculate the magnetostriction and a measurement approach should be applied. In this paper we will only focus on the magnetostriction and the challenges to measure it. A more thorough explanation of the magnetostriction and the measurement methods will be discussed in the next parts.

\section{MAGNETOSTRICTION}

Magnetostriction is a consequence of the magnetization of the ferromagnetic materials. Presenting some basics about the microscopic behaviour of the ferromagnetic materials can help to understand the magnetostriction phenomenon and to indicate the need for a measurement method. The ferromagnetic materials are ordered over volumes containing large numbers (typically $10^{12}-10^{15}$ ) of atoms, called domains. When there is no magnetic field applied, these domains are randomly oriented in different directions and in total the average alignment of all the domains throughout the 
material is zero. In the presence of a magnetic field, when the specimen starts to magnetize, the domains try to align themselves parallel to the field. With a very high magnetic field all the domains are fully aligned with the applied field and the material goes to the saturated state. However below the saturation state, depending on the magnetic field, the domain orientation is not necessarily uniform throughout the material. The behaviour of the magnetostriction of an assembly of domains, a poly-crystal for example, can only be calculated by averaging the effects of domain orientations. Because of the barkhausen effect, which is the sudden discontinuous movements of the domain walls, it is impossible to calculate the magnetostriction strains for a random excitation, and therefore a measurement approach is required [1]. As mentioned before, magnetostriction strongly depends on the applied magnetic field; for a sinusoidal magnetic field, increasing the field results in larger magnetostriction strains. In addition to that, magnetostrictive behaviour of different ferromagnetic materials is not the same. The higher the amount of silicon in the electrical steel, the lower the magnetostriction strains becomes. Also any external pressure to the material when magnetized can alter such behaviour. Magnetostriction is most often demonstrated by measuring the fractional change in length $\Delta L / L$ of a specimen as it is magnetized. $\mathrm{L}$ is the length of the sample and $\Delta L$ is the enlargement or shrinkage of the sample. The order of the magnitude of the deformation of ferromagnetic materials is as small as micrometer per meter. Thus a highly accurate measurement technique is required.

\section{Magnetostriction measurement methods}

In the past, a strain gauge setup was developed in the Electrical Energy Laboratory (EELAB), at Ghent University, Ghent, Belgium. The strain gauge technique is a well known approach. Strain gauges, consisting of a circuit of resistances, are attached on the sample. When the sample is magnetized, the slight deformation of the sample is detected by these resistances. This method is a rather simple method and is often used. However, there are some limitations and drawbacks that will be mentioned here. The accurate attachment of the strain gauges to the sample is very important. When the attachment is not carried out properly, the resistances may not accurately follow the deformation of the specimen, which results in a drop of the accuracy of the strain measurements. Another problem of the strain gauge method is the magnetostriction measurements for low excitations (excitations less than $0.8 \mathrm{~T}$ ), which are usually very difficult to be distinguished from the present noise. The sensitivity of the strain gauges to the presence of high-frequency harmonics is another drawback. A low pass filter can help to solve this problem. The detailed description of the setup is reported in [2] and [3]. A setup using heterodyne laser interferometer was later developed at the EELAB. The lasers, without the need to contact the specimen, measure the vibration of the magnetized specimen. The first design of the setup, the challenges and the step by step improvement, as well as the final design of the setup will be presented in the next paragraph.

\section{Magnetostriction measurement using dual laser heterodyne interferometers}

A new magnetostriction setup using dual heterodyne lasers has been developed at EELAB. Two industrial vibrations sensors (IVS200), calibrated for $5 \mathrm{~mm} / \mathrm{s} / \mathrm{V}$, are used to measure the vibration velocity of a magnetized specimen. The sample preparation, on the contrary to the strain gauge setup, is an easy task. Two pieces of aluminum $(5 \times 5 \mathrm{~mm})$ are glued on a sample with a certain distance. The laser beam are reflected on this aluminum pieces and the vibration signals of those are scattered back to the lasers. The sample with an excitation coil around it is placed between two yokes. The yokes have high magnetic permeability so they provide a closed path for the magnetic flux of the sample. The combination of the specimen, the excitation winding and the yokes are called single sheet tester (SST). In the early design of the setup, this SST was $6 \mathrm{~cm}$ on $8 \mathrm{~cm}$. The two lasers were placed in front of each other and the SST in between with an equal distance from each laser, as shown in Fig 1. The glued mirrors on the sample were 40mm far from each other. In this way, the lasers measured the displacement over the $40 \mathrm{~mm}$ length of the sample. The magnetostriction strains were calculated in a PC program as follows. The lasers actually measure the speed of the mirror vibrations. The first step of the calculation was to add the two velocity signals $v_{1}$ and $v_{2}$, to obtain the total vibrational velocity (see equation1). The length change of the sample was calculated by doing an integration over that total velocity (see equation 2). This value was divided by the original measurement length, in order to have the relative enlargement (see equation3).

$$
v=v_{2}+v_{1}
$$




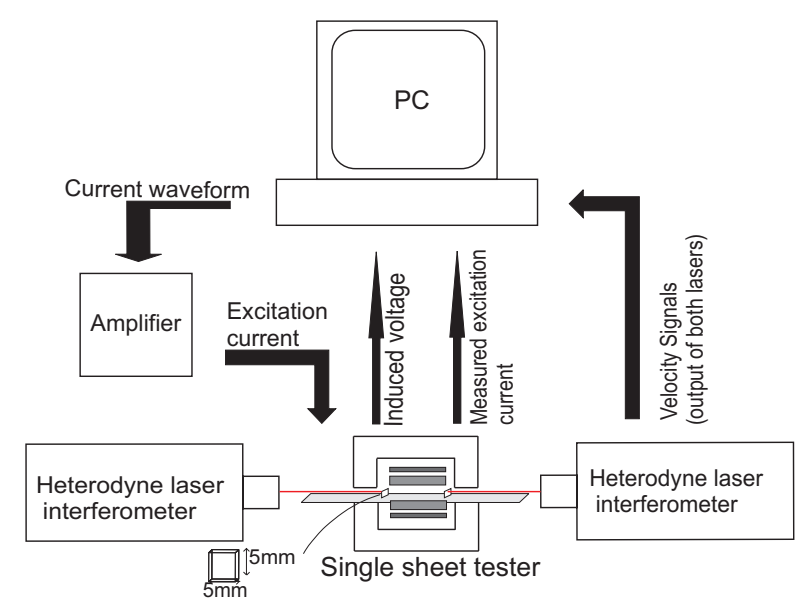

FIGURE 1. The schematic of the early designed magnetostriction measurement setup using dual heterodyne interferometers

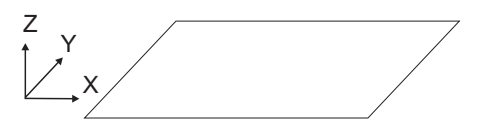

FIGURE 2. The coordinate system

$$
\begin{gathered}
\Delta L=\int v d t \\
\lambda=\frac{\Delta L}{L}
\end{gathered}
$$

The magnetostriction strain is dependent on any external pressure to the sample. To avoid such pressure, a gap of $1 \mathrm{~mm}$ was provided between the sample and the upper yoke by placing small teflon films in each side of the sample. Thus the sample could freely vibrate and deform. However, the measurement results showed low repeatability, especially because of the vibration of the sample in the $\mathrm{z}$ direction (perpendicular plate to the sample) in the gap, for the coordinate system presented in Fig 2. The influence of the tilting of the aluminum mirrors when the sample is magnetized could also play a role in this. On one hand, removing the gap could change the magnetostrictive behaviour of the sample mainly due to factor of the pressure of the top yoke and the possible friction of it with the sample. On the other hand, providing such a gap reduced the measurement accuracy since the sample was displaced a bit when magnetized. To find a compromise, a new measurement approach has been applied. For this new approach, the optical part of setup is designed in a different manner. Instead of the two aluminum mirrors glued on one side of the sample, one is glued on the top and the other one on the bottom of the sample in one place. In this way the lasers measure the vibration of the sample on the both sides. The sample is clamped on the other side between the yokes, easily by closing the gap. The idea is to cancel out the vibration in the $\mathrm{z}$ direction and minimize any possible effect of the mirror tilting. The vibrational velocity of the sample is the average of the two measured velocities:

$$
v=\frac{v_{1}+v_{2}}{2}
$$

The relative displacement of the sample is the integration of this average velocity divided by the length of the sample between the mirrors and the clamped side (see equation 2 and 3 ).

The other modification of this setup was for the SST. As mentioned before, the magnetostriction strains for the ferromagnetic materials are in order of $\mu \mathrm{m} / \mathrm{m}$. So the measurements for a $40 \mathrm{~mm}$ length of the sample resulted in extremely low signals, which could easily be disturbed by the environmental noise. To avoid that, two new bigger yokes of size $17 \mathrm{~cm}$ by $16 \mathrm{~cm}$ were built. The excitation coil and the sample size were also magnified to adapt to the size of the yokes. The measurement distance from the mirrors side to the clamped side of the sample is almost $120 \mathrm{~mm}$, which is 3 times bigger than before. 


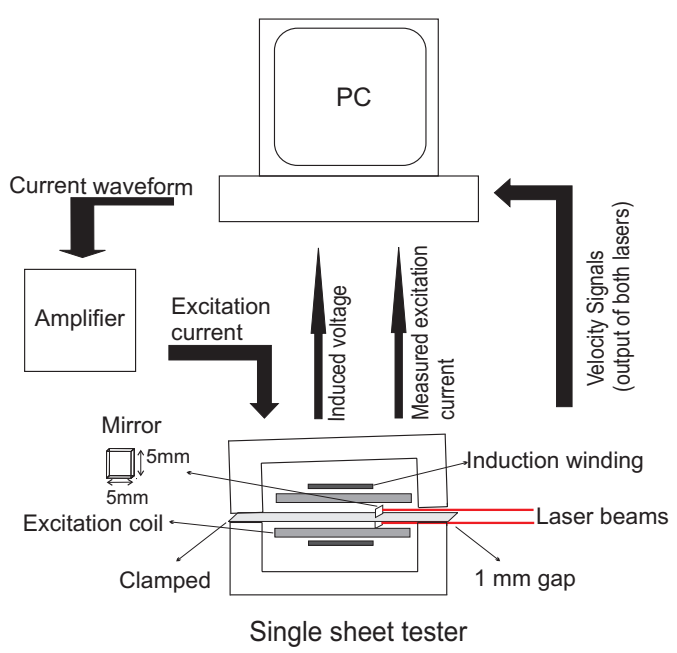

FIGURE 3. The schematic of the new designed magnetostriction measurement setup using dual heterodyne interferometers

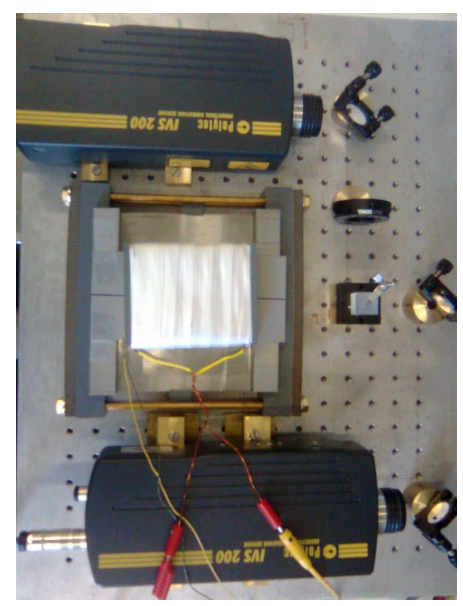

FIGURE 4. The optical part of the setup

Special care is taken to minimalize the influence of external mechanical vibrations on the setup, e.g. noise of the amplifier. To this end, the aluminum setup base frame was replaced by a honey comb table. In order to obtain the maximum reflection back from the aluminum mirrors and also to compensate for the tiny misalignment of these mirrors, retro-reflective tapes were glued on the mirrors. This new setup is presented in Fig3. The honey comb table, the optical components of the setup, and the SST (without the top yoke) are presented in Fig4.

The magnetostriction measurement result with the strain gauge setup and this new-designed laser setup are shown in Fig5 and Fig6. Although the measurement results with the strain gauge approach are filtered for high-frequencies harmonics(harmonics over 40 times the base frequency), still the magnetostrictive behaviour of the sample needs to be detected from the noise. However more filtering can loose part of the data of the magnetostrictive behaviour. The laser measurement results have not been filtered. The influence of the noise on the measurement results by both techniques can be easily observed. The measurements with the laser setup, nicely presents the magnetostrictive behaviour of the material, and no further post processing of the signal is needed. In addition to that, the laser setup is able to measure strains for the excitations around $0.5 \mathrm{~T}$. The sample preparation, as mentioned before, is another advantage of the laser setup over the strain gauge setup. 


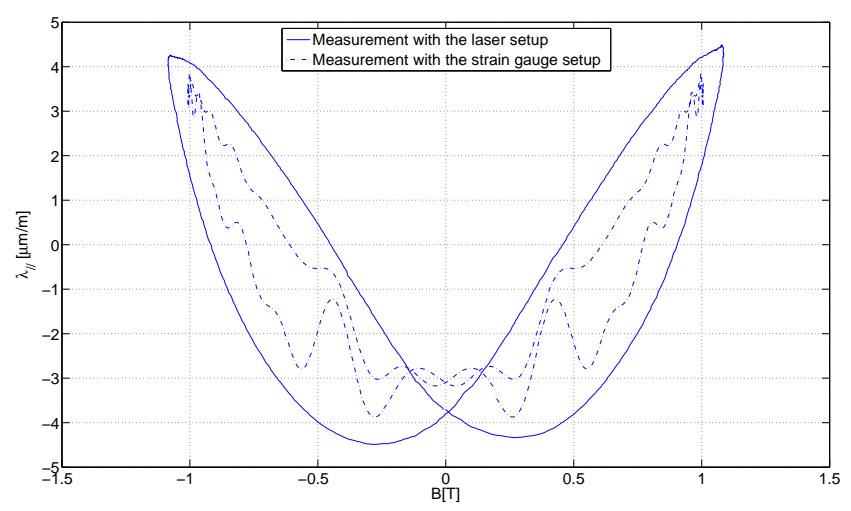

FIGURE 5. Magnetostriction measurements with the strain gauge setup and the new-designed laser setup for a sample without coating under the sinusoidal excitation for $50 \mathrm{~Hz}$ and $\mathrm{B}=1 \mathrm{~T}$

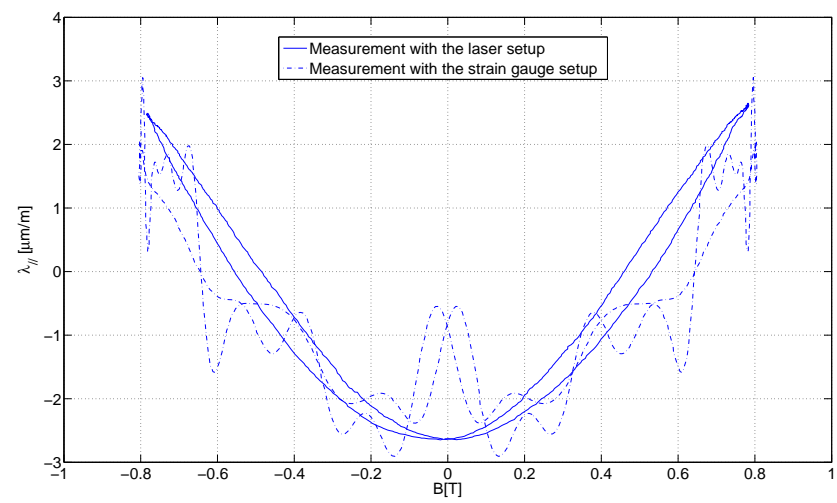

FIGURE 6. Magnetostriction measurements with the strain gauge setup and the new-designed laser setup for a sample without coating under the sinusoidal excitation for $50 \mathrm{~Hz}$ and $\mathrm{B}=0.8 \mathrm{~T}$

\section{CONCLUSION}

A new magnetostriction measurement setup using dual laser heterodyne interferometer has been developed. This new setup and the improvements over time to enhance the accuracy of the measurement are explained. The magnetostriction measurement results with this laser setup are better compared to the strain gauge setup applied before. The main advantages can be summarized as follows. The sample preparation is easier for the laser setup and less time consuming. The magnetostriction measurements for lower excitations than $0.8 \mathrm{~T}$ are possible, with reasonable accuracy. The most noticeable difference is the much lower influence of the noise on the measurement results with the laser method.

\section{REFERENCES}

1. D. Jiles, Introduction to Magnetism and Magnetic Materials, London, Chapman \& Hall, 1991, pp. 98-104

2. T. Hilgert, L. Vandevelde and J. Melkebeek, "Neural-Network-Based Model for Dynamic Hysteresis in the Magnetostriction of Electrical Steel Under Sinusoidal Induction," IEEE Transactions on Magnetics, vol. 43, 2007, pp. 3462-3466

3. T. Hilgert, L. Vandevelde and J. Melkebeek, "Magnetostriction Measurements on Electrical Steels by means of Strain Gauges and Numerical Applications," Przeglad Elektrotechniczny, vol. 81, no. 5, 2005, pp. 87-91 